Bull. Korean Math. Soc. 50 (2013), No. 3, pp. 731-745

http://dx.doi.org/10.4134/BKMS.2013.50.3.731

\title{
SETS AND VALUE SHARING OF $q$-DIFFERENCES OF MEROMORPHIC FUNCTIONS
}

\author{
XiaO-Guang Qi And Lian-Zhong Yang
}

\begin{abstract}
In this paper, we investigate uniqueness problems of certain types of $q$-difference polynomials, which improve some results in [20]. However, our proof is different from that in [20]. Moreover, we obtain a uniqueness result in the case where $q$-differences of two entire functions share values as well. This research also shows that there exist two sets, such that for a zero-order non-constant meromorphic function $f$ and a non-zero complex constant $q, E\left(S_{j}, f\right)=E\left(S_{j}, \Delta_{q} f\right)$ for $j=1,2$ imply $f(z)=t \Delta_{q} f$, where $t^{n}=1$. This gives a partial answer to a question of Gross concerning a zero order meromorphic function $f(z)$ and $\Delta_{q} f$.
\end{abstract}

\section{Introduction}

In what follows, a meromorphic function will always mean meromorphic in the whole complex plane. We say that two meromorphic functions $f$ and $g$ share a value $a \in \mathbb{C} \cup\{\infty\}$ IM (ignoring multiplicities) when $f-a$ and $g-a$ have the same zeros. If $f-a$ and $g-a$ have the same zeros with the same multiplicities, then we say that $f$ and $g$ share the value $a \mathrm{CM}$ (counting multiplicities). We assume that the reader is familiar with the standard symbols and fundamental results of Nevanlinna theory, as found in [12].

As usual, by $S(r, f)$ we denote any quantity satisfying $S(r, f)=o(T(r, f))$ for all $r$ outside of a possible exceptional set of finite linear measure. In particular, we denote by $S_{1}(r, f)$ any quality satisfying $S_{1}(r, f)=o(T(r, f))$ for all $r$ on a set of logarithmic density 1 . Moreover, we denote $\Delta_{q} f=f(q z)-f(z)$, where $q \in \mathbb{C} \backslash\{0,1\}$.

The Nevanlinna theory of $q$-difference expressions and its applications to $q$-difference equations have recently been considered (see $[1,6]$ ). In addition, some results about solutions of zero-order for complex $q$-difference equations, can be found in the introduction in [1].

Applying the $q$-difference analogue of logarithmic derivative lemma, see [1, Theorem 1.1] (Lemma 2.1 in Section 2), Zhang and Korhonen [20] studied the relation between $T(r, f)$ and $T(r, f(q z))$ and concluded $T(r, f(q z))=$

Received February 5, 2012; Revised September 18, 2012.

2010 Mathematics Subject Classification. 30D35, 39A05.

Key words and phrases. meromorphic functions, $Q$-difference, sharing value. 
$(1+o(1)) T(r, f(z))$ on a set of lower logarithmic density 1 , where $f$ is zeroorder meromorphic function. Moreover, They have given an example to show that the zero-order growth restriction in their result above is essentially the best possible, see Remark 1 in [20]. They considered the problem of value distribution and value sharing of $q$-differences polynomials as well. In fact, they got:

Theorem A. Let $f(z)$ and $g(z)$ be two transcendental meromorphic (resp. entire) functions of zero-order. Suppose that $q$ is a non-zero complex constant and $n$ is an integer satisfying $n \geq 8$ (resp. $n \geq 4$ ). If $f(z)^{n} f(q z)$ and $g(z)^{n} g(q z)$ share $1, \infty C M$, then $f(z)=\operatorname{tg}(z)$ for $t^{n+1}=1$.

Theorem B. Let $f(z)$ and $g(z)$ be two transcendental entire functions of zeroorder. Suppose that $q$ is a non-zero complex constant and $n \geq 6$ is an integer. If $f(z)^{n}(f(z)-1) f(q z)$ and $g(z)^{n}(g(z)-1) g(q z)$ share $1 C M$, then $f(z)=g(z)$.

Now it is natural to ask whether $C M$ value sharing can be replaced by $I M$ value sharing in Theorems A and B? The reminder of this paper is organized as follows: Firstly, we give a positive answer to the above question in Section 3 provided the assumption on $n$ is tightened. Then, in Section 4 we prove some uniqueness results for a meromorphic function $f$ sharing some sets with its shift $\Delta_{q} f$, and in Section 5 we obtain some uniqueness results in the case where $q$-differences of two entire functions share values .

\section{Some lemmas}

Lemma 2.1 ([1, Theorem 1.1]). Let $f(z)$ be a zero-order meromorphic function, and $q \in \mathbb{C} \backslash\{0\}$. Then

$$
m\left(r, \frac{f(q z)}{f(z)}\right)=S_{1}(r, f) .
$$

Lemma 2.2 ([20, Theorem 1.1 and Theorem 1.3]). Let $f(z)$ be a zero-order meromorphic function, and $q \in \mathbb{C} \backslash\{0\}$. Then

$$
T(r, f(q z))=(1+o(1)) T(r, f(z))
$$

and

$$
N(r, f(q z))=(1+o(1)) N(r, f(z))
$$

on a set of lower logarithmic density 1.

Lemma 2.3 ([13, Theorem 3.1]). Let $f_{j}(z)(j=1,2,3)$ be meromorphic functions that satisfy

$$
\sum_{j=1}^{3} f_{j}(z) \equiv 1
$$


If $f_{1}(z)$ is not a constant, and

$$
\sum_{j=1}^{3} N_{2}\left(r, \frac{1}{f_{j}}\right)+\sum_{j=1}^{3} \bar{N}\left(r, f_{j}\right)<(\lambda+o(1)) T(r), r \in I,
$$

where $0 \leq \lambda<1, T(r)=\max _{1 \leq j \leq 3}\left\{T\left(r, f_{j}\right)\right\}$, and $I$ has infinite linear measure, then either $f_{2}(z)=1$ or $f_{3}(z)=1$.

Lemma 2.4 ([17, Theorem 1]). Let $f(z)$ and $g(z)$ be two meromorphic functions. If $f(z)$ and $g(z)$ share 1 IM, and if

$$
\limsup _{r \rightarrow \infty} \frac{N^{*}(r, f)+N^{*}(r, g)+N^{*}\left(r, \frac{1}{f}\right)+N^{*}\left(r, \frac{1}{g}\right)}{T(r, f)+T(r, g)}<1,
$$

where $N^{*}(r, f)=2 N_{2}(r, f)+3 \bar{N}(r, f)$ and $r \in I$, then $f(z)=g(z)$ or $f(z) g(z)$ $=1$.

\section{Value sharing of a meromorphic function with its $q$-difference}

Theorem 3.1. Let $f(z)$ and $g(z)$ be two transcendental meromorphic (resp. entire) functions of zero-order. Suppose that $q$ is a non-zero complex constant and $n$ is an integer satisfying $n \geq 26$ (resp. $n \geq 12$ ). If $f(z)^{n} f(q z)$ and $g(z)^{n} g(q z)$ share $1 \mathrm{IM}$, then $f=t_{1} g$ or $f g=t_{2}$, for some constants $t_{1}$ and $t_{2}$ that satisfy $t_{1}^{n+1}=1$ and $t_{2}^{n+1}=1$.

Corollary 3.2. Under the assumptions of Theorem 3.1, and if $f(z)$ and $g(z)$ have at least one pole in common, then $f=t g$ for some constant $t$ that satisfies $t^{n+1}=1$.

Corollary 3.3. Under the assumptions of Theorem 3.1, and if $f(z)$ and $g(z)$ share $\infty I M$, then $f=t g$ for some constant $t$ that satisfies $t^{n+1}=1$.

Theorem 3.4. Let $f(z)$ and $g(z)$ be two transcendental meromorphic functions of zero-order. Suppose that $q$ is a non-zero complex constant such that $|q| \neq 1$ and $n$ is an integer satisfying $n \geq 30$. If $f(z)^{n}(f(z)-1) f(q z)$ and $g(z)^{n}(g(z)-$ 1) $g(q z)$ share $1 I M$, and $f(z)$ and $g(z)$ share $\infty I M$, then $f(z)^{n}(f(z)-$ 1) $f(q z)=g(z)^{n}(g(z)-1) g(q z)$.

Remarks. (1) One might be tempted to want to simplify the conclusion of Theorem 3.4 to conclude $f=g$, as in Theorem B, but the form in Theorem 3.4 may be the best possible. In fact, in the $\mathrm{PhD}$ dissertation of Zhang [19] (in Chinese), he gave a counter-example that

$$
f(z)=\frac{P(z)^{n+1} P(q z)-P(z)}{P(z)^{n+1} P(q z)-1}, \quad g(z)=\frac{P(z)^{n} P(q z)-1}{P(z)^{n+1} P(q z)-1},
$$

where $q$ is a non-zero complex constant and $n$ is a positive integer, $P(z)$ is non-constant entire function of zero-order. By calculation, we get $f(z)^{n}(f(z)-$ 1) $f(q z)=g(z)^{n}(g(z)-1) g(q z)$. Hence $f(z)^{n}(f(z)-1) f(q z)$ and $g(z)^{n}(g(z)-$ 1) $g(q z)$ satisfy the condition of Theorem 3.4 , but $f(z) \neq g(z)$. 
(2) In this section, the assumption that $C M$ is changed into $I M$, provided that at the same time assumption on $n$ is tightened. However, the question of the sharpness of the lower bound for $n$ is inevitable raised, but now we cannot give examples to show the assumptions on $n$ in Theorem 3.1 and Theorem 3.4 are sharp.

Although we cannot get $f(z)=g(z)$ in Theorem 3.4 for meromorphic functions, when $f(z)$ and $g(z)$ are entire, we obtain the following result.

Theorem 3.5. Let $f(z)$ and $g(z)$ be two transcendental entire functions of zero-order. Suppose that $q$ is a non-zero complex constant and $n$ is an integer satisfying $n \geq 16$. If $f(z)^{n}(f(z)-1) f(q z)$ and $g(z)^{n}(g(z)-1) g(q z)$ share 1 $I M$, then $f(z)=g(z)$.

Proof of Theorem 3.1. Let $F(z)=f(z)^{n} f(q z)$ and $G(z)=g(z)^{n} g(q z)$. Thus, $F$ and $G$ share the value $1 I M$.

Case 1. Suppose that $f$ and $g$ are meromorphic. From Lemma 2.2, we get

$$
\begin{aligned}
n T(r, f) & =T\left(r, f^{n}\right)=T\left(r, \frac{F}{f(q z)}\right) \\
& \leq T(r, F)+T(r, f(q z))+S(r, f) \\
& \leq T(r, F)+T(r, f)+S_{1}(r, f),
\end{aligned}
$$

that means

$$
T(r, F) \geq(n-1) T(r, f)+S_{1}(r, f) .
$$

Using the same way, we have

$$
T(r, G) \geq(n-1) T(r, g)+S_{1}(r, g) .
$$

Now we will evaluate the counting functions of $F$ and $G$. From the definition of $F$ and Lemma 2.2, we get

$$
\begin{aligned}
N_{2}\left(r, \frac{1}{F}\right) & \leq 2 \bar{N}\left(r, \frac{1}{f}\right)+N\left(r, \frac{1}{f(q z)}\right)+S(r, f) \\
& \leq 3 T(r, f)+S_{1}(r, f),
\end{aligned}
$$

and

$$
\begin{aligned}
\bar{N}\left(r, \frac{1}{F}\right) & \leq \bar{N}\left(r, \frac{1}{f}\right)+\bar{N}\left(r, \frac{1}{f(q z)}\right)+S(r, f) \\
& \leq 2 T(r, f)+S_{1}(r, f) .
\end{aligned}
$$

Combining (3.3) and (3.4), we know that

$$
N^{*}\left(r, \frac{1}{F}\right) \leq 12 T(r, f)+S_{1}(r, f) .
$$


Similarly, we can get

$$
\begin{aligned}
& N^{*}\left(r, \frac{1}{G}\right) \leq 12 T(r, g)+S_{1}(r, g), \\
& N^{*}(r, F) \leq 12 T(r, f)+S_{1}(r, f), \\
& N^{*}(r, G) \leq 12 T(r, g)+S_{1}(r, g) .
\end{aligned}
$$

Combining (3.5) and (3.6), we know

$$
\begin{aligned}
& N^{*}(r, F)+N^{*}(r, G)+N^{*}\left(r, \frac{1}{F}\right)+N^{*}\left(r, \frac{1}{G}\right) \\
\leq & 24(T(r, f)+T(r, g))+S_{1}(r, f)+S_{1}(r, g) .
\end{aligned}
$$

From (3.1) and (3.2), we get

$$
\frac{N^{*}(r, F)+N^{*}(r, G)+N^{*}\left(r, \frac{1}{F}\right)+N^{*}\left(r, \frac{1}{G}\right)}{T(r, F)+T(r, G)}<1,
$$

when $n \geq 26$. By Lemma 2.4, we can get

$$
F=G \quad \text { or } \quad F G=1 \text {. }
$$

When $F=G$, that is, $f(z)^{n} f(q z)=g(z)^{n} g(q z)$. Let $H(z)=\frac{f(z)}{g(z)}$. Then we have

$$
H(z)^{n}=\frac{1}{H(q z)} .
$$

Assume that $H(z)$ is not a constant, from Lemma 2.2 and (3.7), we have

$$
n T(r, H)=T(r, H(q z))+O(1)=T(r, H)+S_{1}(r, H),
$$

which is a contradiction to $n \geq 26$. Thus, $H(z)$ is a constant, which means that $H^{n+1}=1$. Hence $f=t_{1} g, t_{1}^{n+1}=1$ follows.

When $F G=1$, which implies that

$$
f(z)^{n} f(q z) g(z)^{n} g(q z)=1 .
$$

Set $M(z)=f(z) g(z)$, using the same way as above, we get $M(z)$ must be a constant. From above equation, we obtain $f g=t_{2}$ and $t_{2}^{n+1}=1$.

Case 2. Suppose that $f$ and $g$ are entire. Since now $N^{*}(r, F)=N^{*}(r, G)=$ 0 , furthermore from Lemma 2.1, we get

$$
\begin{aligned}
(n+1) T(r, f) & =T\left(r, f^{n+1}\right)=m\left(r, f^{n+1}\right) \\
& \leq m\left(r, \frac{F(z) f(z)}{f(q z)}\right) \leq m(r, F)+m\left(r, \frac{f(z)}{f(q z)}\right)+S(r, f) \\
& \leq T(r, F)+S_{1}(r, f) .
\end{aligned}
$$

Similarly, we get

$$
T(r, G) \geq(n+1) T(r, g)+S_{1}(r, g) .
$$

Similarly as in the case of meromorphic functions, we get the conclusion, completing the proof of Theorem 3.1. 
Proof of Theorem 3.4. Let

$$
F(z)=f(z)^{n}(f(z)-1) f(q z) \text { and } G(z)=g(z)^{n}(g(z)-1) g(q z) .
$$

Thus, $F$ and $G$ share the value $1 I M$. In the same way as Theorem 3.1, we can get

$$
\begin{aligned}
& T(r, F) \geq n T(r, f)+S_{1}(r, f), \quad T(r, G) \geq n T(r, g)+S_{1}(r, g), \\
& N^{*}\left(r, \frac{1}{F}\right) \leq 17 T(r, f)+S_{1}(r, f), \quad N^{*}\left(r, \frac{1}{G}\right) \leq 17 T(r, g)+S_{1}(r, g), \\
& N^{*}(r, F) \leq 12 T(r, f)+S_{1}(r, f), \quad N^{*}(r, G) \leq 12 T(r, g)+S_{1}(r, g) .
\end{aligned}
$$

By Lemma 2.4, we can get

$$
F=G \quad \text { or } \quad F G=1,
$$

when $n \geq 30$.

In the following, we will prove that the case $F G=1$ does not occur. Otherwise, we assume that $F G=1$, which means

$$
f(z)^{n}(f(z)-1) f(q z) g(z)^{n}(g(z)-1) g(q z)=1 .
$$

Set $h(z)=f(z) g(z)$, then (3.9) can be rewritten as

$$
\left(h(z)^{n+1}-h(z)^{n}(f(z)+g(z))+h(z)^{n}\right) h(q z)=1 .
$$

If $h(z)$ is not a constant. Suppose that there exists a point $z_{0}$ such that $h\left(z_{0}\right)=$ 0 , that is $f\left(z_{0}\right) g\left(z_{0}\right)=0$. From $f$ and $g$ share $\infty I M$, we get $f\left(z_{0}\right) \neq \infty$ and $g\left(z_{0}\right) \neq \infty$. By (3.10), we conclude that $h\left(q z_{0}\right)=\infty$. Hence,

$$
h(z)=0 \Rightarrow h(q z)=\infty .
$$

Next, we suppose that there is a point $z_{1}$ such that $h\left(q z_{1}\right)=0$, from (3.10), we easily get $h\left(z_{1}\right)=\infty$, which means

$$
h(q z)=0 \Rightarrow h(z)=\infty .
$$

Assume $h\left(z_{2}\right)=\infty$, and $z_{2}$ is a pole of $f$ with multiplicity $p$ and a pole of $g$ with multiplicity $q$. Easily, we know $z_{2}$ is a pole of $h(z)^{n+1}, h(z)^{n}(f(z)+g(z))$ with multiplicity $(n+1)(p+q), n(p+q)+\max (p, q)$, respectively. That is, $z_{2}$ is a pole of $\left(h(z)^{n+1}-h(z)^{n}(f(z)+g(z))+h(z)^{n}\right)$ as well. Hence $h\left(q z_{2}\right)=0$, which implies that

$$
h(z)=\infty \Rightarrow h(q z)=0 .
$$

If $h(q z)=\infty \Rightarrow h(z) \neq 0$, then from (3.11) and (3.13)

$$
h(q z)=\infty \Rightarrow h\left(q^{2} z\right)=0 \Rightarrow h\left(q^{3} z\right)=\infty \Rightarrow h\left(q^{2} z\right) \neq 0,
$$

which is impossible. Therefore,

$$
h(q z)=\infty \Rightarrow h(z)=0 .
$$

Case 1. If $|q|<1$, then from (3.11) and (3.13)

$$
h(z)=0 \Rightarrow h(q z)=\infty \Rightarrow h\left(q^{2} z\right)=0 \cdots \Rightarrow h\left(q^{2 k} z\right)=0 \Rightarrow h\left(q^{2 k+1} z\right)=\infty \cdots \text {. }
$$


From above equation, we get

$$
0=\lim _{z \rightarrow 0} h(z)=\infty
$$

which is a contradiction.

Case 2. If $|q|>1$, then from (3.12) and (3.14), we get

$$
h(q z)=0 \Rightarrow h(z)=\infty \Rightarrow h\left(\frac{z}{q}\right)=0 \cdots \Rightarrow h\left(\frac{z}{q^{2 k}}\right)=\infty \Rightarrow h\left(\frac{z}{q^{2 k+1}}\right)=0 \cdots .
$$

From above equation, we also get

$$
0=\lim _{z \rightarrow 0} h(z)=\infty
$$

which is a contradiction.

Therefore, we get $h(z)$ must be a non-zero constant $t$, that is $f g=t$. Since $f$ and $g$ share $\infty I M$, we can easily get $f$ and $g$ have no zeros and no poles. That means the orders of $f$ and $g$ are not less than 1, which contradicts the assumption. Hence, $F G=1$ is not possible, which means $F=G$, that is $f(z)^{n}(f(z)-1) f(q z)=g(z)^{n}(g(z)-1) g(q z)$. We get the conclusion, completing the proof of Theorem 3.4.

Proof of Theorem 3.5. Suppose that $f$ and $g$ are entire. Using the same way as Theorem 3.4 and simple calculations, we get that

$$
f(z)^{n}(f(z)-1) f(q z) g(z)^{n}(g(z)-1) g(q z)=1,
$$

or

$$
f(z)^{n}(f(z)-1) f(q z)=g(z)^{n}(g(z)-1) g(q z) .
$$

when $n \geq 16$.

If $f(z)^{n}(f(z)-1) f(q z) g(z)^{n}(g(z)-1) g(q z)=1$, then we get

$$
N(r, f)=N\left(r, \frac{1}{f}\right)=N\left(r, \frac{1}{f-1}\right)=0,
$$

which is a contradiction.

If $f(z)^{n}(f(z)-1) f(q z)=g(z)^{n}(g(z)-1) g(q z)$, then the assertion now follows the proof in [20, p. 543], we get $f(z)=g(z)$.

\section{Set sharing of a meromorphic function with its $q$-difference}

For a non-constant meromorphic function $f$ and a set $S$ of complex numbers, we define the set $E(S, f)=\bigcup_{a \in S}\{z \mid f(z)-a=0\}$, where a zero of $f-a$ with multiplicity $m$ counts $m$ times in $E(S, f)$. In 1976, Gross asked the following question [3, Question 6]:

Question. Can one find (even one set) finite sets $S_{j}(j=1,2)$ such that any two entire functions $f$ and $g$ satisfying $E\left(S_{j}, f\right)=E\left(S_{j}, g\right)(j=1,2)$ must be identical? 
Since then, many results have been obtained for this and related topics (see $[2,14,15,16]$ ). It is well known that there exists a set $S$ containing seven elements such that if $f$ and $g$ are two non-constant entire functions and $E(S, f)=E(S, g)$, then $f=g$. There are some uniqueness results related to the case when two functions share two sets. We recall the following result given by Gross and Osgood.

Theorem $\mathbf{C}([4])$. Let $S_{1}=\{1,-1\}, S_{2}=\{0\}$. If $f$ and $g$ are non-constant entire functions of finite order such that $E\left(S_{j}, f\right)=E\left(S_{j}, g\right)$ for $j=1,2$, then $f= \pm g$ or $f g=1$.

Many authors considered the condition that removed the order restriction and we just recall the next result for meromorphic functions.

Theorem D ([18]). Let $S_{1}=\left\{1, \omega, \ldots, \omega^{n-1}\right\}$ and $S_{2}=\{\infty\}$, where $\omega=$ $\cos (2 \pi / n)+i \sin (2 \pi / n)$ and $n \geq 6$ be a positive integer. Suppose that $f$ and $g$ are non-constant meromorphic functions such that $E\left(S_{j}, f\right)=E\left(S_{j}, g\right)$ for $j=1,2$, then $f=\operatorname{tg}$ or $f g=t$, where $t^{n}=1$.

If $g$ is replaced by $q$-difference of $f$ in Theorem $\mathrm{D}$, in a recent paper, the present authors considered shared sets problem for $f(z)$ and its $q$-difference $f(q z)$, the result may be stated as follows:

Theorem E ([9]). Let $S_{1}, S_{2}$ be given as in Theorem D. Suppose $f$ is a nonconstant zero-order meromorphic (resp. entire) function such that

$$
E\left(S_{j}, f(z)\right)=E\left(S_{j}, f(q z)\right) \text { for } j=1,2 \text {, and } q \in \mathbb{C} \backslash\{0\} .
$$

If $n \geq 4$ (resp. $n \geq 3)$, then $f(z)=t f(q z), t^{n}=1$ and $|q|=1$.

In this paper, we replace $f(q z)$ with $\Delta_{q} f$, and consider shared sets problem for $f(z)$ and $\Delta_{q} f$. We get the following results:

Theorem 4.1. Let $S_{1}, S_{2}$ be given as in Theorem D. Suppose $f$ is a nonconstant zero-order meromorphic function such that $E\left(S_{j}, f(z)\right)=E\left(S_{j}, \Delta_{q} f\right)$ for $j=1,2$, and $q \in \mathbb{C} \backslash\{0\}$. If $n \geq 5$, then $f(z)=t \Delta_{q} f$, where $t^{n}=1$.

Using the same proof of Theorem 4.1, we get:

Corollary 4.2. Theorem 4.1 still holds if $f$ is a non-constant zero-order entire function and $n \geq 3$.

Corresponding to Corollary 4.2, it is natural to consider the case $n=2$. The following result is a partial answer as to what may happen if $n=2$ in Corollary 4.2 .

Theorem 4.3. Let $f$ be a non-constant zero-order entire function and $q \in$ $\mathbb{C} \backslash\{0\}$. If $f(z)$ and $\Delta_{q} f$ share the set $\{a, b\} C M$, where $a, b$ are two distinct constants, then $f(z)=\Delta_{q} f$.

Corollary 4.4. Under the condition of Theorem 4.3, if $f(z)$ and $\Delta_{q} f$ share the set $\{1,-1\} C M$, then $f(z)=\Delta_{q} f$. 
Remarks. (1) Let $f(z)=z+3$ and $q=-1$. Then $f(q z)=f(-z)=-z+3$ and $\Delta_{q} f=-2 z$. We get $f(z)$ and $\Delta_{q} f$ share $2 C M$, however, the conclusion of Theorem 4.3 does not hold. This example shows that Theorem 4.3 cannot hold when the sharing set contains only one element, which means the assumption of Theorem 4.3 is sharp.

(2) From Corollary 4.2 and Corollary 4.4, we obtain that Theorem 4.1 still holds if $f$ is a non-constant zero-order entire function and $n \geq 2$. Furthermore, the assumption $n \geq 2$ is sharp by the example in Remark (1).

(3) Let $f(z)=z$ and $q=2$. Then $f(q z)=f(2 z)=2 z$ and $\Delta_{q} f=z$. Easily, we get that $f(z)$ and $\Delta_{q} f$ share $\{1,-1\} C M$. This implies that in this section, we cannot get $|q|=1$ as in Theorem E.

Proof of Theorem 4.1. The main idea of this proof is from Theorem E, while the details are somewhat different. For the convenience of the reader, we give a complete proof here. From the assumption of Theorem 4.1, we know $f(z)^{n}$ and $\left(\Delta_{q} f\right)^{n}$ share 1 and $\infty C M$, we obtain that

$$
\frac{\left(\Delta_{q} f\right)^{n}-1}{f(z)^{n}-1}=C,
$$

where $C$ is a non-zero constant. Rewrite (4.1) as

$$
\left(\Delta_{q} f\right)^{n}=C\left(f(z)^{n}-1+\frac{1}{C}\right) .
$$

Set

$$
H(z)=\frac{f(z)^{n}}{1-\frac{1}{C}} .
$$

If $C \not \equiv 1$, then we apply the second main theorem to $H(z)$, and get

$$
\begin{aligned}
n T(r, f)+S(r, f) & =T(r, H) \\
& \leq \bar{N}\left(r, \frac{1}{H}\right)+\bar{N}(r, H)+\bar{N}\left(r, \frac{1}{H-1}\right)+S(r, H) \\
& \leq \bar{N}\left(r, \frac{1}{f}\right)+\bar{N}(r, f)+\bar{N}\left(r, \frac{1}{f(z)^{n}-1+\frac{1}{C}}\right)+S(r, f) \\
& \leq \bar{N}\left(r, \frac{1}{f}\right)+\bar{N}(r, f)+\bar{N}\left(r, \frac{1}{\Delta_{q} f}\right)+S(r, f) \\
& \leq 2 T(r, f)+T(r, f(q z)-f(z))+S(r, f) .
\end{aligned}
$$

Combining (4.3) with Lemma 2.2, we get

$$
n T(r, f) \leq 4 T(r, f)+S_{1}(r, f),
$$

which contradicts $n \geq 5$. Therefore, $C \equiv 1$, that is, $f(z)^{n}=\left(\Delta_{q} f\right)^{n}$, so we have $f(z)=t \Delta_{q} f$, for a constant $t$ with $t^{n}=1$. 
Proof of Corollary 4.2. Using a similar way of Theorem 4.1, equation (4.3) follows. By the assumption that $f(z)$ is entire, we have

$$
\begin{aligned}
n T(r, f) & \leq T(r, f)+m(r, f(q z)-f(z))+S(r, f), \\
& \leq T(r, f)+m(r, f)+m\left(r, \frac{f(q z)-f(z)}{f(z)}\right)+S(r, f) .
\end{aligned}
$$

From the above equation, Lemma 2.1 and Lemma 2.2, we get

$$
n T(r, f) \leq 2 T(r, f)+S_{1}(r, f),
$$

which contradicts $n \geq 3$. The assertion now follows as in Theorem 4.1.

Proof of Theorem 4.3. Set

$$
F(z)=f(z)-\frac{a+b}{2}, G(z)=\Delta_{q} f-\frac{a+b}{2} .
$$

From the assumption that $f(z)$ and $\Delta_{q} f$ share $\{a, b\}$ CM, we obtain that $F(z)$ and $G(z)$ share $\{d,-d\}$ CM, where $d=\frac{a-b}{2}$. As $a$ and $b$ are distinct, we know $d$ is a non-zero constant. Since $f(z)$ is an entire function of zero-order it follows that

$$
\frac{(G(z)-d)(G(z)+d)}{(F(z)-d)(F(z)+d)}=\frac{\left(\Delta_{q} f-a\right)\left(\Delta_{q} f-b\right)}{(f-a)(f-b)}=C^{2},
$$

where $C$ is a non-zero constant.

Case 1. $C^{2} \equiv 1$. From (4.4), we have

$$
(f(q z)-a-b)\left(\Delta_{q} f-f(z)\right)=0,
$$

which implies that $\Delta_{q} f=f(z)$, we get the conclusion.

Case 2. $C^{2} \not \equiv 1$. Let $h_{1}(z)=F(z)-\frac{1}{C} G(z), h_{2}(z)=F(z)+\frac{1}{C} G(z)$, then

$$
F(z)=\frac{1}{2}\left(h_{1}+h_{2}\right), \quad G(z)=\frac{C}{2}\left(h_{2}-h_{1}\right) .
$$

And from (4.4), we get

$$
h_{1} h_{2}=\left(1-\frac{1}{C^{2}}\right) d^{2}
$$

From equation (4.6) and the condition $d$ is non-zero constant, we get

$$
N\left(r, \frac{1}{h_{1}}\right)=0, \quad N\left(r, \frac{1}{h_{2}}\right)=0 .
$$

Combining (4.7) with the fact that $h_{1}(z)$ and $h_{2}(z)$ are zero-order entire functions, we obtain $h_{1}$ and $h_{2}$ are non-zero constant. From (4.5), we get $f(z)$ is a constant, which contradicts the assumption. Hence, only Case 1 of Theorem 4.3 holds, we get the conclusion. 


\section{Value sharing by $q$-difference of entire functions}

The classical results due to Nevanlinna [7] in the uniqueness theory of meromorphic functions are the five-point, resp. four-point, theorems:

Theorem F. If two non-constant meromorphic functions $f(z)$ and $g(z)$ share five distinct values $a_{1}, a_{2}, a_{3}, a_{4}, a_{5} \in \mathbb{C} \cup\{\infty\}$ IM, then $f \equiv g$.

Theorem G. If two non-constant meromorphic functions $f(z)$ and $g(z)$ share four distinct values $a_{1}, a_{2}, a_{3}, a_{4} \in \mathbb{C} \cup\{\infty\} C M$, then $f \equiv g$ or $f \equiv T \circ g$, where $T$ is a Möbius transformation.

In [8], we considered the uniqueness problem in the case when $f(z)$ shares values with $f(q z)$ for a zero-order meromorphic function $f(z)$. We got the following results:

Theorem H. Let $f(z)$ be a non-constant zero-order meromorphic function, and $q \in \mathbb{C} \backslash\{0\}$, and let $a_{1}, a_{2}, a_{3} \in \mathbb{C} \cup\{\infty\}$ be three distinct values. If $f(z)$ and $f(q z)$ share $a_{1}, a_{2} C M$ and $a_{3} I M$, then $f(z)=f(q z)$ and $|q|=1$.

Theorem I. Let $f(z)$ be a non-constant zero-order entire function, $q \in \mathbb{C} \backslash\{0\}$, and let $a_{1}, a_{2} \in \mathbb{C}$ be two distinct values. If $f(z)$ and $f(q z)$ share $a_{1}$ and $a_{2}$ IM, then $f(z)=f(q z)$ and $|q|=1$.

As applications of Theorem $\mathrm{H}$ and Theorem I, we get the following two results:

Theorem 5.1. Let $f(z)$ and $g(z)$ be two meromorphic functions with $f(z)$ of zero-order, $q \in \mathbb{C} \backslash\{0\}$, and let $a_{1}, a_{2}, a_{3} \in \mathbb{C} \cup\{\infty\}$ be three distinct values. Suppose that $f(z)$ and $g(z)$ share $a_{1}, a_{2} C M$ and $a_{3}$ IM. If $f(z)=f(q z)$, then $g(z)=g(q z)$, and $|q|=1$.

Theorem 5.2. Let $f(z)$ and $g(z)$ be two entire functions with $f(z)$ of zeroorder, $q \in \mathbb{C} \backslash\{0\}$, and let $a_{1}, a_{2} \in \mathbb{C}$ be two distinct values. Suppose that $f(z)$ and $g(z)$ share $a_{1}$ and $a_{2}$ IM. If $f(z)=f(q z)$, then $g(z)=g(q z)$, and $|q|=1$.

In 1976, Yang [11] proposed the following problem.

Suppose that $f(z)$ and $g(z)$ are two transcendental entire functions such that $f(z)$ and $g(z)$ share $0 C M$ and $f^{\prime}$ and $g^{\prime}$ share $1 C M$. What can be said about the relationship between $f(z)$ and $g(z)$ ?

Shibazaki [10] proved the following result.

Theorem K. Suppose that $f(z)$ and $g(z)$ are entire functions of finite order such that $f^{\prime}$ and $g^{\prime}$ share $1 C M$. If $\delta(0, f) \geq 0$ and 0 is a Picard value of $g(z)$, then either $f(z)=g(z)$ or $f^{\prime} g^{\prime}=1$. $\mathrm{K}$.

The following result can be seen as a $q$-difference counterpart to Theorem

Theorem 5.3. Suppose that $f(z)$ and $g(z)$ are two non-constant entire functions of zero-order, and let $a$ and $b$ be distinct constants such that $\delta(a)=$ 
$\delta(a, f)+\delta(a, g)>1$. If $f\left(q_{1} z\right)$ and $g\left(q_{2} z\right)$ share $b C M$, then $f(z)=g(q z)$, where $q=\frac{q_{2}}{q_{1}}$ and $q_{1}, q_{2}$ are non-zero complex constants.

Proof of Theorem 5.1. From the proof of Theorem H, we obtain that $f(z)$ and $g(z)$ share $0, \infty \mathrm{CM}$ and 1 IM by some transformations. By the second main theorem, we know

$$
\begin{aligned}
T(r, g) & \leq \bar{N}(r, g)+\bar{N}\left(r, \frac{1}{g}\right)+\bar{N}\left(r, \frac{1}{g-1}\right)+S(r, g) \\
& \leq \bar{N}(r, f)+\bar{N}\left(r, \frac{1}{f}\right)+\bar{N}\left(r, \frac{1}{f-1}\right)+S(r, g) \\
& \leq 3 T(r, f)+S(r, g) .
\end{aligned}
$$

Similarly,

$$
T(r, f) \leq 3 T(r, g)+S(r, f) .
$$

Combining the above two estimates, we obtain $\sigma(f)=\sigma(g)=0$, where $\sigma(f)$ is the order of $f(z)$. Since $f(z)$ and $g(z)$ share $0, \infty \mathrm{CM}$, and $f(z)$ and $g(z)$ are zero-order, it follows that

and

$$
\frac{f(z)}{g(z)}=C
$$

$$
\frac{f(q z)}{g(q z)}=C .
$$

From the above two equations and the condition that $f(z)=f(q z)$, we deduce that $g(z)=g(q z)$. Using the same way of Theorem H, we get $|q|=1$. This completes the proof of Theorem 5.1.

Proof of Theorem 5.2. Using a similar proof of Theorem 5.1, we conclude that $\sigma(f)=\sigma(g)=0$ as well. By the assumption, we know, if there exists a point $z_{0}$ such that $g\left(z_{0}\right)=a_{i}$, then $f\left(z_{0}\right)=a_{i}$, where $i=1,2$. As $f(z)=f(q z)$, we get $f\left(q z_{0}\right)=a_{i}$, hence $g\left(q z_{0}\right)=a_{i}$. Similarly, if $g\left(q z_{1}\right)=a_{i}$, we can obtain $g\left(z_{1}\right)=a_{i}$. Therefore, we get that zero-order entire functions $g(z)$ and $g(q z)$ share $a_{1}$ and $a_{2}$ IM. By Theorem I, we know $g(z)=g(q z)$, where $|q|=1$.

Proof of Theorem 5.3. The former part of Theorem 5.3 follows by using the same reasoning as in [5] with apparent modification. For the convenience of the reader, we give a complete proof.

From $\delta(a)>1$, we can easily get $\delta(a, f)>0$ and $\delta(a, g)>0$. Now we take a positive number $\varepsilon$ such that $(2+2 \varepsilon-\delta(a))<1, \delta(a, f)-\varepsilon>0$ and $\delta(a, g)-\varepsilon>0$. Then we have

$$
(\delta(a, f)-\varepsilon) T(r, f) \leq m\left(r, \frac{1}{f-a}\right)
$$

and

$$
(\delta(a, g)-\varepsilon) T(r, g) \leq m\left(r, \frac{1}{g-a}\right)
$$


as $r \rightarrow \infty$. By Lemma 2.1, we deduce that

$$
m\left(r, f\left(q_{1} z\right)\right) \leq m(r, f)+S_{1}(r, f),
$$

and

$$
m\left(r, \frac{1}{f-a}\right) \leq m\left(r, \frac{1}{f\left(q_{1} z\right)-a}\right)+S_{1}(r, f) .
$$

Set

$$
F(z)=\frac{f\left(q_{1} z\right)-a}{b-a}, \quad G(z)=\frac{g\left(q_{2} z\right)-a}{b-a} .
$$

From (5.1), (5.3)-(5.5), we get

$$
\begin{aligned}
(\delta(a, f)-\varepsilon) T(r, f) & \leq m\left(r, \frac{1}{f\left(q_{1} z\right)-a}\right)+S_{1}(r, f) \\
& \leq T\left(r, f\left(q_{1} z\right)\right)+S_{1}(r, f) \\
& \leq T(r, F)+S_{1}(r, f) \leq T(r, f)+S_{1}(r, f) .
\end{aligned}
$$

Similarly,

$$
(\delta(a, g)-\varepsilon) T(r, g) \leq T(r, G)+S_{1}(r, g) \leq T(r, g)+S_{1}(r, g) .
$$

Hence

$$
S_{1}(r, F)=S_{1}(r, f), \quad S_{1}(r, G)=S_{1}(r, g) .
$$

Again from (5.1) and (5.4), we obtain that

$$
\begin{aligned}
(\delta(a, f)-\varepsilon) T(r, F) & \leq(\delta(a, f)-\varepsilon) T(r, f)+S_{1}(r, f) \\
& \leq m\left(r, \frac{1}{f\left(q_{1} z\right)-a}\right)+S_{1}(r, f) \\
& \leq T(r, F)-N\left(r, \frac{1}{F}\right)+S_{1}(r, f) .
\end{aligned}
$$

So we have

$$
N\left(r, \frac{1}{F}\right) \leq(1-\delta(a, f)+\varepsilon) T(r, F)
$$

By the same reasoning, we get

$$
N\left(r, \frac{1}{G}\right) \leq(1-\delta(a, g)+\varepsilon) T(r, G)
$$

Since $f\left(q_{1} z\right)$ and $g\left(q_{2} z\right)$ share $b \mathrm{CM}$, we obtain that,

$$
\frac{f\left(q_{1} z\right)-b}{g\left(q_{2} z\right)-b}=C
$$

where $C$ is a non-zero constant. From (5.11) we have

$$
F(z)-G(z) C+C \equiv 1 .
$$

Set $F_{1}(z)=F(z), F_{2}(z)=G(z) C, F_{3}(z)=C$. Then

$$
F_{1}+F_{2}+F_{3}=1 \text {, }
$$


and

$$
T(r)=\max _{1 \leq j \leq 3}\left\{T\left(r, F_{j}\right)\right\}, \quad S(r)=o(T(r)) .
$$

From (5.9) and (5.10), we get

$$
\begin{aligned}
\sum_{j=1}^{3} N_{2}\left(r, \frac{1}{F_{j}}\right)+\sum_{j=1}^{3} \bar{N}\left(r, F_{j}\right) & \leq N\left(r, \frac{1}{F}\right)+N\left(r, \frac{1}{G}\right)+S(r) \\
& \leq(2+2 \varepsilon-\delta(a)) T(r)+S(r) .
\end{aligned}
$$

By Lemma 2.3, we get that $F_{2}=1$ or $F_{3}=1$. If $F_{2}=1$, then we get $G(z)$ is a constant, which is a contradiction; while if $F_{3}=1$, the conclusion holds.

Acknowledgements. The authors thank the referee for his/her valuable suggestions to improve the present paper. This work was supported by the NSFC Tianyuan Mathematics Youth Fund (No. 11226094), the NSF of Shandong Province, China (No. ZR2012AQ020 and No. ZR2010AM030) and the Fund of Doctoral Program Research of University of Jinan (XBS1211).

\section{References}

[1] D. C. Barnett, R. G. Halburd, R. J. Korhonen, and W. Morgan, Nevanlinna theory for the q-difference operator and meromorphic solutions of q-difference equations, Proc. Roy. Soc. Edinburgh Sect. A 137 (2007), no. 3, 457-474.

[2] G. Frank and M. Reinders, A unique range set for meromorphic functions with 11 elements, Complex Var. Theory Appl. 37 (1998), no. 1-4, 185-193.

[3] F. Gross, Factorization of meromorphic functions and some open problems, Complex analysis (Proc. Conf., Univ. Kentucky, Lexington, Ky., 1976), pp. 51-67. Lecture Notes in Math., Vol. 599, Springer, Berlin, 1977.

[4] F. Gross and C. F. Osgood, Entire functions with common preimages, in: Factorization theory of meromorphic functions and related topics, pp. 19-24, Lecture Notes in Pure and Appl. Math., 78, Dekker, New York,

[5] X. H. Hua, A unicity theorem for entire functions, Bull. London Math. Soc. 22 (1990), no. $5,457-462$.

[6] I. Laine and C. C. Yang, Clunie theorem for difference and q-difference polynomials, J. London. Math. Soc. (2) 76 (2007), no. 3, 556-566.

[7] R. Nevanlinna, Le théorème de Picard-Borel et la théorie des fonctions méromorphes, Gauthiers-Villars, Paris, 1929.

[8] X. G. Qi, K. Liu, and L. Z. Yang, Value sharing results of a meromorphic function $f(z)$ and $f(q z)$, Bull. Korean. Math. Soc. 48 (2011), no. 6, 1235-1243.

[9] X. G. Qi and L. Z. Yang, Share sets of q-difference of meromorphic functions, to appear in Math. Slovaca.

[10] K. Shibazaki, Unicity theorem for entire functions of finite order, Mem. Nat. Defense Acad. (Japan) 21 (1981), no. 3, 67-71.

[11] C. C. Yang, On two entire functions which together with their first derivative have the same zeros, J. Math. Anal. Appl. 56 (1976), no. 1, 1-6.

[12] C. C. Yang and H. X. Yi, Uniqueness Theory of Meromorphic Functions, Kluwer Academic Publishers, 2003.

[13] L. Z. Yang and J. L. Zhang, Non-existence of meromorphic solution of a Fermat type functional equation, Aequationes Math. 76 (2008), no. 1-2, 140-150.

[14] H. X. Yi, A question of Gross and the uniqueness of entire functions, Nagoya Math. J. 138 (1995), 169-177. 
[15] _ Unicity theorems for meromorphic or entire functions II, Bull. Austral. Math. Soc. 52 (1995), no. 2, 215-224.

[16] Unicity theorems for meromorphic or entire functions III, Bull. Austral. Math. Soc. 53 (1996), no. 1, 71-82.

[17] _ Meromorphic functions that share one or two values II, Kodai Math. J. 22, (1999), no. 2, 264-272.

[18] H. X. Yi and L. Z. Yang, Meromorphic functions that share two sets, Kodai Math. J. 20 (1997), no. 2, 127-134.

[19] X. B. Zhang, Uniqueness of meromorphic functions sharing a small function and some normality criteria, Doctoral Dissertation, Shandong University, 2011.

[20] J. L. Zhang and R. Korhonen, On the Nevanlinna characteristic of $f(q z)$ and its applications, J. Math. Anal. Appl. 369 (2010), no. 2, 537-544.

XiaO-GuANG Qi

UNIVERSITY OF JINAN

SCHOOL OF MATHEMATICS

Jinan, Shandong, 250022, P. R. China

E-mail address: xiaogqi@gmail.com or xiaogqi@mail.sdu.edu.cn

LIAN-ZHONG YANG

Shandong University, School of Mathematics

Jinan, Shandong, 250100, P. R. China

E-mail address: lzyang@sdu.edu.cn 